September 25, 2008

\title{
The Kerr/CFT Correspondence
}

\author{
Monica Guica ${ }^{\dagger}$, Thomas Hartman ${ }^{\S}$, Wei Song ${ }^{\S \ddagger}$, and Andrew Strominger ${ }^{\S}$ \\ ${ }^{\dagger}$ LPTHE, Université Pierre et Marie Curie-Paris 6; CNRS \\ Boîte 126, 4 Pl. Jussieu, 75252 Paris Cedex 05, France \\ $\S$ Center for the Fundamental Laws of Nature \\ Jefferson Physical Laboratory, Harvard University, Cambridge, MA 02138, USA
}

\begin{abstract}
Quantum gravity in the region very near the horizon of an extreme Kerr black hole (whose angular momentum and mass are related by $J=G M^{2}$ ) is considered. It is shown that consistent boundary conditions exist, for which the asymptotic symmetry generators form one copy of the Virasoro algebra with central charge $c_{L}=\frac{12 J}{\hbar}$. This implies that the near-horizon quantum states can be identified with those of (a chiral half of) a two-dimensional conformal field theory (CFT). Moreover, in the extreme limit, the Frolov-Thorne vacuum state reduces to a thermal density matrix with dimensionless temperature $T_{L}=\frac{1}{2 \pi}$ and conjugate energy given by the zero mode generator, $L_{0}$, of the Virasoro algebra. Assuming unitarity, the Cardy formula then gives a microscopic entropy $S_{\text {micro }}=\frac{2 \pi J}{\hbar}$ for the CFT, which reproduces the macroscopic Bekenstein-Hawking entropy $S_{\text {macro }}=\frac{\text { Area }}{4 \hbar G}$. The results apply to any consistent unitary quantum theory of gravity with a Kerr solution. We accordingly conjecture that extreme Kerr black holes are holographically dual to a chiral two-dimensional conformal field theory with central charge $c_{L}=\frac{12 J}{\hbar}$, and in particular that the near-extreme black hole GRS $1915+105$ is approximately dual to a CFT with $c_{L} \sim 2 \times 10^{79}$.
\end{abstract}

$\ddagger$ On leave from the Institute of Theoretical Physics, Academia Sinica, Beijing 100080, China 


\section{Contents}

1 Introduction $\quad 1$

2 Kerr review

3 The NHEK geometry

4 The Asymptotic Symmetry Group 66

5 Boundary Conditions $\quad 7$

6 Generators $\quad 9$

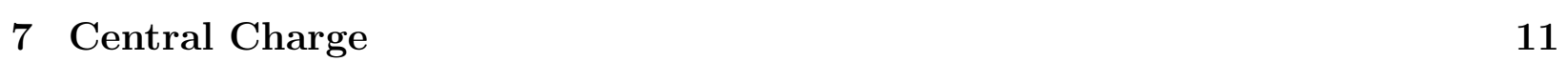

\begin{tabular}{ll|l|}
8 & Temperature & 12 \\
\hline
\end{tabular}

9 Microscopic origin of the Bekenstein-Hawking-Kerr entropy

A Asymptotic constraints

\begin{tabular}{|l|l|} 
B Charge integrability & 15 \\
\hline
\end{tabular}

\section{Introduction}

One of the deepest discoveries in modern theoretical physics is that of holographic dualities, which relate a quantum theory of gravity to a quantum field theory without gravity in fewer dimensions. These dualities become especially powerful when combined with string theory [1]. It is an occasional misconception, however, that the existence of holographic dualities is contingent on the validity of string theory. This is not the case. For example, the demonstration [2] that any consistent theory of quantum gravity on three-dimensional anti-de Sitter space $\left(\mathrm{AdS}_{3}\right)$ is holographically dual to a two-dimensional conformal field theory (CFT) did not invoke string theory. When holographic duality was used to find the microscopic origin of the Bekenstein-Hawking entropy for a class of black holes, the construction at first appeared to depend heavily on details of string theory [3]. However, it was later understood [4] to apply to essentially any consistent, unitary quantum theory of gravity containing the black holes as classical solutions. In the last few years we are beginning to see interesting applications of holographic duality outside of string theory in nuclear [5, 6, 7], condensed matter [8, 9, 10] and atomic [11, 12] physics. 
Oddly, the rich ideas surrounding holographic dualities so far have not been successfully applied to the enigmatic objects which largely inspired their original discovery - the Schwarzschild or Kerr black holes we actually observe in the sky 11 In this paper we attempt to fill this gap by arguing, in the spirit of [2, 4], that extreme Kerr black holes are holographically dual to a chiral CFT in two dimensions. An extreme Kerr black hole is one for which the angular momentum $J$ saturates the bound $J \leq G M^{2}$. More angular momentum with the same mass $M$ leads to a violation of cosmic censorship. Nearly extreme black holes have been seen in the sky. For example GRS 1915+105, with mass $M \sim 14 M_{\text {sun }}$, has $J / G M^{2}>0.98$ [13], and corrections to the dual CFT representation of GRS 1915+105 should be correspondingly suppressed. In addition, at extremality the ISCO (the innermost stable circular orbit on the accretion disc) coincides with the event horizon, so near extremality the ISCO is within the near-horizon region. Therefore the observed emissions from the ISCO should be well-described by the dual CFT2 2 It is our hope that the rich experimental [13, 16] and theoretical [17] literature on Kerr black holes can be illuminated by the dual CFT description.

Our argument that Kerr is dual to a CFT parallels the general one given by Brown and Henneaux [2] for $\mathrm{AdS}_{3}$, except that we replace $\mathrm{AdS}_{3}$ with the NHEK (near-horizon extreme Kerr) geometry found by Bardeen and Horowitz [18] via a near-horizon limiting procedure 3 Despite having different dimensions, the spaces bear some resemblance: a slice of NHEK at a particular fixed polar angle is a discrete quotient of $\mathrm{AdS}_{3}$. We first carefully specify boundary conditions at the asymptotic infinity of NHEK (which is where, before taking the near-horizon limit, it is joined to Minkowski space in the full Kerr solution) and demonstrate their consistency. We then show that, given these boundary conditions, the so-called asymptotic symmetry group (ASG) is one copy of the conformal group and furthermore has a central charge $c_{f}=\frac{12 J}{\hbar}$. Hence extreme Kerr, with the given boundary conditions, is dual to a chiral CFT, 4

While this very general analysis gives the central charge of the dual CFT, it tells us little else about the detailed structure of the CFT. For that to be determined we would need an

\footnotetext{
${ }^{1}$ The successes so far have mainly concerned black holes with large amounts of charge and/or in dimensions other than four.

${ }^{2}$ In [14, 15] greybody scattering factors for various black holes were computed using the dual CFT picture, and found to agree with those computed by conventional methods. Computations of this type may also be possible for Kerr, and generalized to the context of accretion discs.

${ }^{3}$ In this procedure the asymptotically flat region, whose excitations we do not regard as part of the black hole itself, is excised and one is left only with the portion of the spacetime neighboring the black hole horizon.

${ }^{4} \mathrm{We}$ do not have an argument for modular invariance and are not distinguishing here between the chiral sector of a nonchiral CFT and a CFT with only a chiral sector. It is interesting to note however that a necessary condition for the partition function of the latter to be modular invariant up to a sign, accounting for the presence of fermions, given $c=12 J / \hbar$ is precisely that $J / \hbar$ is half-integral.
} 
ultraviolet completion (for example string theory) of quantum gravity on the Kerr background. However the information about the central charge, together with the assumption of unitarity, turns out to be exactly enough to compute the extreme Kerr entropy by counting quantum microstates, as in [4]. An analysis of the extreme limit of the Frolov-Thorne vacuum, which generalizes the Hartle-Hawking vacuum for Schwarzchild to Kerr, shows that the CFT must be at temperature $T_{L}=\frac{1}{2 \pi}$. We then apply the thermodynamic Cardy formula relating the microscopic entropy of a unitary CFT to its temperature and central charge. The resulting entropy agrees exactly with the macroscopic Bekenstein-Hawking area-entropy law, providing corroboration for our proposal that extreme Kerr is dual to a two-dimensional chiral CFT.

The fact that we encounter only a chiral half of a CFT ultimately derives from the fact that at extremality the rotational velocity of the Kerr horizon becomes the speed of light. Hence both edges of the forward light cone coincide as the horizon is approached and force all physical excitations(such as the edge of the accretion disc), which must lie between the edges of light cone, to spin around chirally with the black hole. Away from extremality this is no longer the case and we may expect to encounter a non-chiral CFT. This very interesting but difficult problem will not be considered herein.

We wish to stress that, while mere consistency imposes very strong constraints, we have not analyzed all possibilities and have not shown that our near-horizon boundary conditions are the unique consistent choice for studying extreme Kerr. While we did not find any other consistent and nontrivial choices, our search was not exhaustive, and there may well be others with different consequences. Ultimately, the appropriate boundary conditions should be determined from the physical question. We do suspect that weaker or different boundary conditions will be needed for the just-mentioned problem of near-extremal excitations. These issues remain for future work.

Section 2 reviews the Kerr geometry and section 3 its near-horizon limit. In section 4 we review the notion of an ASG. Our boundary conditions are specified in section 5 , and the generators $L_{n}$ of the corresponding ASG are shown to form a Virasoro algebra in section 6 . The central charge is computed in section 7. In section 8 we take the limit of the FrolovThorne vacuum for Kerr, and show that it yields a thermal state with temperature $\frac{1}{2 \pi}$. In the concluding section we microscopically compute the entropy for extreme Kerr from the Cardy formula and find that it reproduces the macroscopic Bekenstein-Hawking area law. Some technical points are relegated to two appendices.

Previous work on a dual description of Kerr, some in the context of string theory, includes [14, 19, 20, 21]. 


\section{Kerr review}

The Kerr metric [22, 23] is the general rotating black hole solution of the four-dimensional vacuum Einstein equations. In Boyer-Lindquist coordinates it is

$$
\begin{gathered}
d s^{2}=-\frac{\Delta}{\rho^{2}}\left(d \hat{t}-a \sin ^{2} \theta d \hat{\phi}\right)^{2}+\frac{\sin ^{2} \theta}{\rho^{2}}\left(\left(\hat{r}^{2}+a^{2}\right) d \hat{\phi}-a d \hat{t}\right)^{2}+\frac{\rho^{2}}{\Delta} d \hat{r}^{2}+\rho^{2} d \theta^{2} \\
\Delta \equiv \hat{r}^{2}-2 M r+a^{2}, \quad \rho^{2} \equiv \hat{r}^{2}+a^{2} \cos ^{2} \theta \\
a \equiv \frac{G J}{M}, \quad M \equiv G M_{A D M}
\end{gathered}
$$

It is labeled by two parameters: the angular momentum $J$ and the geometric mass $M$. In order to simplify the formulae, but at the risk of some confusion, in the above and hereafter we have rescaled $M$ by a factor of $G$ relative to the abstract and introduction. The solution has naked singularities unless $J$ lies in the parameter range

$$
\frac{-M^{2}}{G} \leq J \leq \frac{M^{2}}{G}
$$

Of course, quantum mechanically $J$ is quantized

$$
J=\hbar j
$$

for some half integer $j$. There is an event horizon at

$$
r_{+}=M+\sqrt{M^{2}-a^{2}}
$$

The Hawking temperature, surface gravity and angular velocity of the horizon are

$$
\begin{gathered}
T_{H}=\frac{\hbar \kappa}{2 \pi}=\frac{\hbar\left(r_{+}-M\right)}{4 \pi M r_{+}}, \\
\Omega_{H}=\frac{a}{2 M r_{+}} .
\end{gathered}
$$

These are related by the first law to the Bekenstein-Hawking entropy [24, 25]

$$
S_{B H}=\frac{\text { Area }}{4 \hbar G}=\frac{2 \pi M r_{+}}{\hbar G} .
$$

We are primarily interested in the so-called extreme Kerr, which carries the maximum allowed angular momentum

$$
J=\frac{M^{2}}{G}
$$


Extreme Kerr has zero Hawking temperature but a nonzero entropy

$$
S_{B H}=\frac{2 \pi J}{\hbar}
$$

Our goal is to to explain this number as the logarithm of the number of quantum microstates of Kerr.

\section{The NHEK geometry}

We wish to study the region very near the extreme Kerr horizon at $\hat{r}=M$. In order to do so, following Bardeen and Horowitz [18] we define, new (dimensionless) coordinates

$$
t=\frac{\lambda \hat{t}}{2 M}, \quad y=\frac{\lambda M}{\hat{r}-M}, \quad \phi=\hat{\phi}-\frac{\hat{t}}{2 M}
$$

and take $\lambda \rightarrow 0$ keeping $(t, y, \phi, \theta)$ fixed. The result is the near-horizon extreme Kerr or "NHEK" geometry in Poincaré-type coordinates

$$
d s^{2}=2 G J \Omega^{2}\left(\frac{-d t^{2}+d y^{2}}{y^{2}}+d \theta^{2}+\Lambda^{2}\left(d \phi+\frac{d t}{y}\right)^{2}\right)
$$

where

$$
\Omega^{2} \equiv \frac{1+\cos ^{2} \theta}{2}, \quad \Lambda \equiv \frac{2 \sin \theta}{1+\cos ^{2} \theta}
$$

$\phi \sim \phi+2 \pi$ and $0 \leq \theta \leq \pi$. The NHEK geometry is not asymptotically flat. Note that the angular momentum affects only the overall scale of the geometry.

The coordinates (3.2) cover only part of the NHEK geometry. Global coordinates $(r, \tau, \varphi)$ are given by (for a discussion of global properties see [18])

$$
\begin{aligned}
y & =\left(\cos \tau \sqrt{1+r^{2}}+r\right)^{-1} \\
t & =y \sin \tau \sqrt{1+r^{2}} \\
\phi & =\varphi+\ln \left(\frac{\cos \tau+r \sin \tau}{1+\sin \tau \sqrt{1+r^{2}}}\right) .
\end{aligned}
$$

The metric (3.2) is then

$$
d \bar{s}^{2}=2 G J \Omega^{2}\left(-\left(1+r^{2}\right) d \tau^{2}+\frac{d r^{2}}{1+r^{2}}+d \theta^{2}+\Lambda^{2}(d \varphi+r d \tau)^{2}\right) .
$$


The NHEK geometry has an enhanced $S L(2, \mathbb{R}) \times U(1)$ isometry group [18]. The rotational $U(1)$ isometry is generated by the Killing vector

$$
\zeta_{0}=-\partial_{\varphi}
$$

Time translations become part of an enhanced $S L(2, \mathbb{R})$ isometry group generated by the Killing vectors

$$
\begin{aligned}
& \tilde{J}_{1}=2 \sin \tau \frac{r}{\sqrt{1+r^{2}}} \partial_{\tau}-2 \cos \tau \sqrt{1+r^{2}} \partial_{r}+\frac{2 \sin \tau}{\sqrt{1+r^{2}}} \partial_{\varphi} \\
& \tilde{J}_{2}=-2 \cos \tau \frac{r}{\sqrt{1+r^{2}}} \partial_{\tau}-2 \sin \tau \sqrt{1+r^{2}} \partial_{r}-\frac{2 \cos \tau}{\sqrt{1+r^{2}}} \partial_{\varphi} \\
& \tilde{J}_{0}=2 \partial_{\tau}
\end{aligned}
$$

Note that all of these isometries act within a three-dimensional slice of fixed polar angle $\theta$. The geometry of these slices is a quotient of warped $\mathrm{AdS}_{3}$ (the $\mathrm{AdS}_{3}$ analog of the squashed $\mathrm{S}^{3}$ ), with the quotient arising from the $\varphi$ identification [26, 27]. Such quotients are (warped) black holes, much as $\mathrm{AdS}_{3}$ quotients are BTZ black holes [28]. The $\tau, r$ plane describes $\mathrm{AdS}_{2}$, while the $\varphi$ circle is an $S^{1}$ bundle over the $\mathrm{AdS}_{2}$. At the special value of $\theta$ where $\Omega^{2}=\sin \theta$, the slice is locally an ordinary $\mathrm{AdS}_{3}$ and acquires a local $S L(2, \mathbb{R})_{R} \times S L(2, \mathbb{R})_{L}$ isometry. At all other values of $\theta$, the $S L(2, \mathbb{R})_{L}$ is broken to $\mathrm{U}(1)$. Near the equator we have a "stretched" $\mathrm{AdS}_{3}$ quotient (as the $S^{1}$ fiber is stretched), while near the poles we have a "squashed" $\mathrm{AdS}_{3}$ quotient. Properties of these three-dimensional spacetimes in a context relevant to the present one were recently described in [28].

\section{The Asymptotic Symmetry Group}

We now turn to the study of excitations around near-horizon extreme Kerr. This requires imposing boundary conditions at the $S^{2} \times \mathbb{R}$ boundary $y=0$. Since we lost the asymptotically flat region in taking the near-horizon limit, this boundary is not flat and it is not a priori obvious what boundary conditions we should use. Indeed, different boundary conditions may be relevant in different physical contexts. For every consistent set of boundary conditions there is an associated asymptotic symmetry group (ASG). This is defined as the set of allowed symmetry transformations modulo the set of trivial symmetry transformations

$$
\text { ASG }=\frac{\text { Allowed Symmetry Transformations }}{\text { Trivial Symmetry Transformations }}
$$

Here 'allowed' means that the transformation is consistent with the specified boundary conditions, while 'trivial' means that the generator of the transformation vanishes after we have implemented the constraints and reduced it to a boundary integral. 
Consistency requires that the generators of the ASG be well defined and not diverge at the boundary. If the boundary conditions are too strong, all interesting excitations are ruled out. If they are too weak, the generators of the ASG are ill-defined. In general, there is a narrow window of consistent boundary conditions. For example, in asymptotically flat space, one usually requires that excitations of the metric fall off like $\frac{1}{r}$ or faster at infinity. The ASG is then simply the Poincaré group. One might try to demand that the metric fall off spatially as $\frac{1}{r^{2}}$. This would allow only zero energy configurations and hence the theory would be trivial. On the other hand, one might try to demand that it fall off as $\frac{1}{\sqrt{r}}$. Then the energy and other symmetry generators would be in general divergent, and it is unlikely any sense could be made of the theory. So the general idea is to make the falloff weak enough to include the physics of interest, while still maintaining finiteness of the generators.

\section{Boundary Conditions}

We choose the boundary conditions

$$
\left(\begin{array}{cccc}
h_{\tau \tau}=\mathcal{O}\left(r^{2}\right) & h_{\tau \varphi}=\mathcal{O}(1) & h_{\tau \theta}=\mathcal{O}\left(\frac{1}{r}\right) & h_{\tau r}=\mathcal{O}\left(\frac{1}{r^{2}}\right) \\
h_{\varphi \tau}=h_{\tau \varphi} & h_{\varphi \varphi}=O(1) & h_{\varphi \theta}=\mathcal{O}\left(\frac{1}{r}\right) & h_{\varphi r}=\mathcal{O}\left(\frac{1}{r}\right) \\
h_{\theta \tau}=h_{\tau \theta} & h_{\theta \varphi}=h_{\varphi \theta} & h_{\theta \theta}=\mathcal{O}\left(\frac{1}{r}\right) & h_{\theta r}=\mathcal{O}\left(\frac{1}{r^{2}}\right) \\
h_{r \tau}=h_{\tau r} & h_{r \varphi}=h_{\varphi r} & h_{r \theta}=h_{\theta r} & h_{r r}=\mathcal{O}\left(\frac{1}{r^{3}}\right)
\end{array}\right),
$$

where $h_{\mu \nu}$ is the deviation of the full metric from the background NHEK metric $\bar{g}$ in (3.7). We note that the allowed deviations $h_{\tau \tau}$ and $h_{\varphi 4} 5$ are of the same order as the leading terms in (3.7). In this regard, these boundary conditions differ for example from the usual $\mathrm{AdS}_{3}$ boundary conditions [2], where all deviations are subleading. An analysis with a number of similarities to the present one (with non-subleading deviations ) for the BMS group at $\mathcal{I}^{+}$ can be found in [29, 30]. The most general diffeomorphisms which preserve the boundary conditions (5.1) are of the form

$$
\xi=\left[-r \epsilon^{\prime}(\varphi)+O(1)\right] \partial_{r}+\left[C+O\left(\frac{1}{r^{3}}\right)\right] \partial_{\tau}+\left[\epsilon(\varphi)+O\left(\frac{1}{r^{2}}\right)\right] \partial_{\varphi}+O\left(\frac{1}{r}\right) \partial_{\theta}
$$

where $\epsilon(\varphi)$ is an arbitrary smooth function of the boundary coordinate $\varphi$, and $\mathrm{C}$ is an arbitrary constant. The subleading terms indicated above can be seen, after computing the generators, to correspond to trivial diffeomorphisms. Therefore the asymptotic symmetry group contains one copy of the conformal group of the circle generated by 6

$$
\zeta_{\epsilon}=\epsilon(\varphi) \partial_{\varphi}-r \epsilon^{\prime}(\varphi) \partial_{r}
$$

\footnotetext{
${ }^{5}$ The asymptotic constraints force a linear combination of these, the trace of $h_{\mu \nu}$, to vanish at linear order, as described in appendix A.

${ }^{6} \zeta_{\epsilon}$ is discontinuous at the north and south poles $\theta=(0, \pi)$. This can be regulated by taking for example $\tilde{\zeta}_{\epsilon}=\frac{r^{2} \sin \theta}{1+r^{2} \sin \theta}\left[\epsilon(\varphi) \partial_{\varphi}-r \epsilon^{\prime}(\varphi) \partial_{r}\right]$. Expanding in $\frac{1}{r}$ we see that $\tilde{\zeta}_{\epsilon}$ and $\zeta_{\epsilon}$ differ by trivial diffeomorphisms, while $\tilde{\zeta}_{\epsilon}$ is smooth for any finite $r$.
} 
This Virasoro algebra here has only a $U(1)$, not an $S L(2, \mathbb{R})$, isometry subgroup 7 The NHEK metric (3.2) transforms under (5.3) as

$$
\delta_{\epsilon} d \bar{s}^{2}=4 J G \Omega^{2}\left(r^{2}\left(1-\Lambda^{2}\right) \partial_{\varphi} \epsilon d \tau^{2}-\frac{r \partial_{\varphi}^{2} \epsilon}{1+r^{2}} d \varphi d r+\Lambda^{2} \partial_{\varphi} \epsilon d \varphi^{2}-\frac{\partial_{\varphi} \epsilon}{\left(1+r^{2}\right)^{2}} d r^{2}\right)
$$

Since $\varphi \sim \varphi+2 \pi$ (because $\phi \sim \phi+2 \pi$ ), it is convenient to define $\epsilon_{n}(\varphi)=-e^{-i n \varphi}$ and $\zeta_{n}=\zeta\left(\epsilon_{n}\right)$. Under Lie brackets, these symmetry generators obey the Virasoro algebra

$$
i\left[\zeta_{m}, \zeta_{n}\right]_{L . B .}=(m-n) \zeta_{m+n}
$$

Note that $\zeta_{0}$ generates the $U(1)$ rotational isometry.

The allowed symmetry transformations (5.2) also include $\tau$ translations generated by $\partial_{\tau}$. The conjugate conserved quantity, which we denoted $E_{R}$, measures the deviation $\frac{M^{2}}{G}-J$ of the black hole from extremality. Here we wish to study only the extremal black holes, which entails a restriction to the subspace in which $E_{R}$ vanishes. This restriction should be compatible with (5.3) because $\partial_{\tau}$ commutes with the Virasoro generators. It can be implemented with an additional boundary condition, given in section 6 below, which makes the generator of $\tau$ translations trivial.

The reader may wonder how we came up with the boundary conditions (5.1). We began by assuming (a) the existence of a non-trivial Virasoro whose zero mode is proportional to $\partial_{\varphi}$ in the allowed diffeomorphisms, (b) the boundary conditions can be linearly described in terms of power law falloff of the individual components of the metric fluctuations. We found only one self-consistent set of boundary conditions with these properties, up to possible further constraints on subleading terms which do not affect the ASG or its central charge. In studies of the Gödel black hole [31] and warped $\mathrm{AdS}_{3}$ [32], consistent boundary conditions were imposed in which the $S L(2, \mathbb{R})$ isometry is enhanced to a Virasoro algebra, and the $U(1)$ isometry is enhanced to a current algebra. That is quite different than the situation here (as well as in [33]) in which the $S L(2, \mathbb{R})$ becomes trivial and the $U(1)$ is enhanced to a Virasoro and therefore do not meet requirement (a) above. We expect that consistent boundary conditions analogous to those described in [31, 32] do exist for Kerr. If so, they are likely relevant to an understanding of the entropy of near-extremal fluctuations (since the $\bar{L}_{0}$ of the $S L(2, \mathbb{R})$ measures the deviation from extremality) rather than the ground state entropy of extreme Kerr.

\footnotetext{
${ }^{7}$ This suggests that the CFT state dual to the Kerr vacuum is not $S L(2, \mathbb{R})$ invariant.
} 


\section{Generators}

Now we need to construct the surface integrals which generate the diffeomorphisms of (5.3) via Dirac brackets, and see if they are finite. When the deviations $h$ of the metric are not subleading, the charges can have nonlinear corrections, which must be carefully considered. For this purpose the covariant formalism of Barnich, Brandt and Compère [34, 35], based on [36, 37, 38, 39, 40, 41] and further developed in [42, 43], is the most complete and will be adopted in the following. An example, mathematically quite similar to the present one, are the Gödel black holes analyzed in [31].

The generator of a diffeomorphism $\zeta$ is a conserved charge $Q_{\zeta}[g] .8$ Under Dirac brackets, the charges associated with asymptotic symmetries obey the same algebra as the symmetries themselves, up to a possible central term. Infinitesimal charge differences between neighboring geometries $g_{\mu \nu}$ and $g_{\mu \nu}+h_{\mu \nu}$ are given by

$$
\delta Q_{\zeta}[g]=\frac{1}{8 \pi G} \int_{\partial \Sigma} k_{\zeta}[h, g]
$$

where the integral is over the boundary of a spatial slice and

$$
\begin{aligned}
k_{\zeta}[h, g]= & -\frac{1}{4} \epsilon_{\alpha \beta \mu \nu}\left[\zeta^{\nu} D^{\mu} h-\zeta^{\nu} D_{\sigma} h^{\mu \sigma}+\zeta_{\sigma} D^{\nu} h^{\mu \sigma}+\frac{1}{2} h D^{\nu} \zeta^{\mu}\right. \\
& \left.-h^{\nu \sigma} D_{\sigma} \zeta^{\mu}+\frac{1}{2} h^{\sigma \nu}\left(D^{\mu} \zeta_{\sigma}+D_{\sigma} \zeta^{\mu}\right)\right] d x^{\alpha} \wedge d x^{\beta} .
\end{aligned}
$$

Covariant derivatives and raised indices are computed using $g_{\mu \nu}$. In asymptotically AdS spacetimes, the formula (6.1) for the charge is true even for finite $h$, and it agrees with the charges obtained in the classic Hamiltonian [2, 44, 45] or quasilocal [46, 47] formalisms. However, in certain cases such as 5d Gödel spacetimes [48, 49], nonlinear contributions are important near the boundary, and only infinitesimal $h$ is allowed. In those cases, finite charge differences are computed by integrating $\delta Q$ over a path in the configuration space,

$$
Q_{\zeta}[g]-Q_{\zeta}[\bar{g}]=\int_{\gamma} \delta Q_{\zeta}[g(\gamma)]
$$

where $\gamma$ connects $\bar{g}$ to $g$ and $h(\gamma)$ in (6.1) is taken tangent to the path. Path-independence holds provided certain integrability conditions are satisfied [35, 43]. We show that these conditions are obeyed around NHEK in appendix B.

The charges that generate $\partial_{\tau}$ and $\zeta_{\epsilon}$ are

$$
Q_{\partial_{\tau}}=\frac{1}{8 \pi G} \int_{\partial \Sigma} k_{\partial_{\tau}}, \quad Q_{\zeta_{\epsilon}}=\frac{1}{8 \pi G} \int_{\partial \Sigma} k_{\zeta_{\epsilon}} .
$$

\footnotetext{
${ }^{8}$ We choose the arbitrary additive constants appearing in [34, 35] so that $Q_{\zeta}[\bar{g}]=0$ for $\bar{g}$ the NHEK metric.
} 
Choosing $g_{\mu \nu}$ to be the NHEK background, the integrands simplify to

$$
\begin{aligned}
k_{\partial_{\tau}}= & -\left(\frac{1}{4 \Lambda} r\left[\left(\Lambda^{4}+\Lambda^{2}-2\right) h_{\varphi \varphi}+\frac{\Lambda^{4}}{r^{2}} h_{t t}\right]\right. \\
& -\frac{1}{4 \Lambda}\left[r^{3} \Lambda^{4} h_{r r}+2 r^{2} \Lambda \partial_{\theta}\left(\Lambda h_{r \theta}\right)+2 \Lambda^{2} r \partial_{\tau} h_{r \varphi}+2\left(\Lambda^{2}-1\right) r^{2} \partial_{r} h_{\varphi \varphi}\right. \\
& \left.\left.+2 \Lambda^{4} h_{\tau \varphi}-\Lambda^{2} r\left(\Lambda^{2}-2+2 r \partial_{r}\right) h_{\theta \theta}\right]\right) d \theta \wedge d \phi+\cdots \\
k_{\zeta_{\epsilon}}= & \frac{1}{4 \Lambda}\left[2 \Lambda^{2} \epsilon^{\prime} r h_{r \varphi}-\epsilon \Lambda^{2}\left(\Lambda^{2} \frac{h_{\tau \tau}}{r^{2}}+\left(\Lambda^{2}+1\right) h_{\varphi \varphi}+2 r \partial_{\varphi} h_{r \varphi}\right)\right] d \theta \wedge d \varphi+\cdots
\end{aligned}
$$

We have assumed the boundary conditions (5.1) and discarded total $\varphi$ derivatives. The $+\cdots$ includes terms which vanish for $r \rightarrow \infty$ or are not tangent to $\partial \Sigma$, and so do not contribute to the integral. From the boundary conditions (5.1) we see immediately that $k_{\zeta_{\epsilon}}$, and therefore $Q_{\zeta_{\epsilon}}$, are finite around NHEK. For a general background $g_{\mu \nu}$, a straightforward counting of powers of $r$ term by term in (6.2) reveals that $Q_{\zeta_{\epsilon}}$ remains finite.

In addition, we must show that $Q_{\partial_{\tau}}$, which measures the deviation from extremality, is well-defined. This does not follow immediately from the boundary conditions (5.1 $)^{9}$. In fact, as we are studying extreme Kerr, we want this charge not only to be finite, but to vanish altogether, i.e. to be trivial. We therefore impose the supplementary boundary condition

$$
E_{R} \equiv Q_{\partial_{\tau}}[g]=0
$$

This is equivalent to requiring that the pullback of $k_{\partial_{\tau}}$ to the boundary obeys $\left.k_{\partial_{\tau}}\right|_{\partial \Sigma}=\left.d X\right|_{\partial \Sigma}$ for some one-form $X$ globally defined on $\partial \Sigma$. Under the constraint (6.7), only perturbations $h$ which preserve (6.7) and only background metrics $g$ which can be reached from the NHEK geometry via a path of such perturbations are considered. This is presumably a complicated nonlinear submanifold of the geometries allowed by the linear boundary conditions (5.1). It can be shown that the $E_{R}=0$ submanifold contains in particular finite generalizations of the infinitesmal $\zeta_{\epsilon}$ diffeomorphisms acting on the NHEK geometry 10 These carry nonzero $Q_{\zeta_{\epsilon}}$ charges. The inclusion of such spaces is expected because the $\zeta_{\epsilon}$ and $\partial_{\tau}$ commute. We do not know if there are other types of spaces with $E_{R}=0$. The answer likely depends on the matter content of the theory, about which so far we have assumed only that it does not affect the boundary behavior.

It remains to be seen that, with the supplementary boundary condition (6.7), the transformations $\zeta_{\epsilon}$ are still allowed. Formally this follows from the fact that $\zeta_{\epsilon}$ and $\partial_{\tau}$ commute, but we must be careful about divergences. It is easy to check directly that the perturbation

\footnotetext{
${ }^{9} \mathrm{~A}$ similar structure was encountered in 31 , who similarly impose a supplementary boundary condition.

${ }^{10}$ Verifying this by explicit computation is a bit tricky because of subtleties at the north and south pole, and uses the fact that $d k_{\partial_{\tau}}=0$ on shell [34]. To make the computation well defined, one must use a regulated form of $\zeta_{\epsilon}$ as e.g. given in footnote 6 .
} 
(5.4), which results from the action of $\zeta_{\epsilon}$ on the NHEK geometry, indeed yields a $k_{\partial_{\tau}}$ obeying (6.7). For the more general background consistent with (6.7), we use the fact that the generators $Q_{\zeta_{\epsilon}}$ are well defined on the bigger space of geometries obeying only (5.1). Therefore, they properly generate the local action of a $\zeta_{\epsilon}$ diffeomorphism. This will preserve the local expression $\left.k_{\partial_{\tau}}\right|_{\partial \Sigma}=\left.d X\right|_{\partial \Sigma}$ of $k_{\partial_{\tau}}$ as an exact form on $\partial \Sigma$ up to a c-number corresponding to a possible central term. The central term is [34]

$$
\frac{1}{8 \pi G} \int_{\partial \Sigma} k_{\zeta_{\epsilon}}\left[\mathcal{L}_{\tau} \bar{g}, \bar{g}\right]
$$

where $\mathcal{L}_{\tau}$ is the Lie derivative along $\tau$. As there is no possible central term between the generators of Virasoro and $\tau$ translations, this must vanish, in agreement with explicit computation. Therefore we can consistently restrict to extremal configurations by imposing (6.7).

\section{Central Charge}

The Dirac bracket algebra of the asymptotic symmetry group is computed by varying the charges

$$
\left\{Q_{\zeta_{m}}, Q_{\zeta_{n}}\right\}_{D . B .}=Q_{\left[\zeta_{m}, \zeta_{n}\right]}+\frac{1}{8 \pi G} \int_{\partial \Sigma} k_{\zeta_{m}}\left[\mathcal{L}_{\zeta_{n}} \bar{g}, \bar{g}\right]
$$

For the NHEK geometry the Lie derivative gives

$$
\begin{aligned}
\mathcal{L}_{\zeta_{n}} \bar{g}_{\tau \tau} & =4 G J \Omega^{2}\left(1-\Lambda^{2}\right) r^{2} i n e^{-i n \varphi} \\
\mathcal{L}_{\zeta_{n}} \bar{g}_{r \varphi} & =-\frac{2 G J \Omega^{2} r}{1+r^{2}} n^{2} e^{-i n \varphi} \\
\mathcal{L}_{\zeta_{n}} \bar{g}_{\varphi \varphi} & =4 G J \Lambda^{2} \Omega^{2} i n e^{-i n \varphi} \\
\mathcal{L}_{\zeta_{n}} \bar{g}_{r r} & =-\frac{4 G J \Omega^{2}}{\left(1+r^{2}\right)^{2}} i n e^{-i n \varphi}
\end{aligned}
$$

It follows that

$$
\frac{1}{8 \pi G} \int_{\partial \Sigma} k_{\zeta_{m}}\left[\mathcal{L}_{\zeta_{n}} \bar{g}, \bar{g}\right]=-i\left(m^{3}+2 m\right) \delta_{m+n} J
$$

Let us now define dimensionless quantum versions of the $Q \mathrm{~s}$ by

$$
\hbar L_{n} \equiv Q_{\zeta_{n}}+\frac{3 J}{2} \delta_{n}
$$

plus the usual rule of Dirac brackets to commutators as $\{., .\}_{D . B .} \rightarrow-\frac{i}{\hbar}[.,$.$] . The quantum$ charge algebra is then

$$
\left[L_{m}, L_{n}\right]=(m-n) L_{m+n}+\frac{J}{\hbar} m\left(m^{2}-1\right) \delta_{m+n, 0} .
$$


From this we can read off the central charge for extreme Kerr

$$
c_{L}=\frac{12 J}{\hbar} \text {. }
$$

For GRS $1915+105$, this gives $c_{L}=(2 \pm 1) \times 10^{79}$, with the uncertainty coming from the uncertainty in the measured mass.

We note that (7.9) does not depend on the details of the boundary conditions (5.1) in that it holds for any boundary conditions as long as the diffeomorphisms (5.3) are allowed.

\section{Temperature}

In this section we derive the relation $T_{L}=\frac{1}{2 \pi}$ for the generalized temperature of the nearhorizon region in units of its inverse radius.

First, we must define the quantum vacuum for extreme Kerr. This problem i s subtle because Kerr has no everywhere timelike Killing vector, so in fact, globally, there is no quantum state with all the desired properties of a vacuum. There is an extensive literature on this subject for the generic Kerr black hole, references to which can be found in [50]. Frolov and Thorne [51] define a vacuum by using a Killing vector field which is timelike from the horizon out to the speed of light surface, which is the surface at which an observer must move at the speed of light in order to corotate with the black hole. The Frolov-Thorne vacuum has some pathologies outside of this surface [52], but is well behaved in the nearhorizon region [50], where it is an analog of the Hartle-Hawking vacuum for Schwarzschild and is therefore ideal for our purposes.

Construction of the Frolov-Thorne vacuum for generic Kerr begins by expanding the quantum fields in eigenmodes of the asymptotic energy $\omega$ and angular momentum $m$. For example for a scalar field $\Phi$ we may write

$$
\Phi=\sum_{\omega, m, l} \phi_{\omega m l} e^{-i \omega \hat{t}+i m \hat{\phi}} f_{l}(r, \theta) .
$$

After tracing over the region inside the horizon, the vacuum is a diagonal density matrix in the energy-angular momentum eigenbasis with a Boltzmann weighting factor

$$
e^{-\hbar \frac{\omega-\Omega_{H} m}{T_{H}}} .
$$

This reduces to the Hartle-Hawking vacuum in the non-rotating $\Omega_{H}=0$ case.

In order to transform this to near-horizon quantities and take the extremal limit (in which $T_{H} \rightarrow 0$ ) we note that in the near-horizon coordinates

$$
e^{-i \omega \hat{t}+i m \hat{\phi}}=e^{-\frac{i}{\lambda}(2 M \omega-m) t+i m \phi}=e^{-i n_{R} t+i n_{L} \phi},
$$


where

$$
n_{L} \equiv m, \quad n_{R} \equiv \frac{1}{\lambda}(2 M \omega-m)
$$

are the left and right charges associated to $\partial_{\phi}$ and $\partial_{t}$ in the near-horizon region. In terms of these variables the Boltzmann factor (8.2) is

$$
e^{-\hbar \frac{\omega-\Omega_{H} m}{T_{H}}}=e^{-\frac{n_{L}}{T_{L}}-\frac{n_{R}}{T_{R}}},
$$

where the dimensionless left and right temperatures are

$$
T_{L}=\frac{r_{+}-M}{2 \pi\left(r_{+}-a\right)}, \quad T_{R}=\frac{r_{+}-M}{2 \pi \lambda r_{+}} .
$$

In the extremal limit $M^{2} \rightarrow G J$ these reduce to

$$
T_{L}=\frac{1}{2 \pi}, \quad T_{R}=0 .
$$

The left-movers are then thermally populated with the Boltzmann distribution at temperature $1 / 2 \pi \cdot 11$

$$
e^{-2 \pi n_{L}}
$$

Note that even though extreme Kerr has zero Hawking temperature, the quantum fields outside the horizon are not in a pure state.

\section{Microscopic origin of the Bekenstein-Hawking-Kerr entropy}

In the previous section we saw that the quantum theory in the Frolov-Thorne vacuum restricted to extreme Kerr has the left-moving temperature

$$
T_{L}=\frac{1}{2 \pi}
$$

Since the states of quantum gravity on NHEK, with the boundary conditions (5.1), are identified under the holographic duality with those of the left-moving part of the CFT, the CFT dual of the Frolov-Thorne vacuum must also have temperature (9.1). The central charge of the CFT was shown to be

$$
c_{L}=\frac{12 J}{\hbar}
$$

\footnotetext{
${ }^{11} \mathrm{~A}$ fast but less rigorous way to derive this result is to note that at every fixed polar angle $\theta$, the geometry is a quotient of warped $\mathrm{AdS}_{3}$. The temperature for such quotients is the length of the shift determining the quotient divided by $4 \pi^{2}[28,53]$. This gives $T_{L}=\frac{1}{2 \pi}$ for every $\theta$.
} 
According to the Cardy formula the entropy for a unitary CFT at large $T_{L}$ obey 12

$$
S=\frac{\pi^{2}}{3} c_{L} T_{L}
$$

Using (9.1),(9.2), we find the microscopic entropy for the dual to extreme Kerr

$$
S_{\text {micro }}=\frac{2 \pi J}{\hbar}=S_{B H} .
$$

This exactly reproduces the macroscopic Bekenstein-Hawking entropy (2.11) of the extreme Kerr black hole.

\section{Acknowledgements}

This work was supported in part by DOE grant DE-FG02-91ER40654. We are grateful to Dionysios Anninos, Geoffrey Compère, Allison Farmer, Valeri Frolov, Gary Horowitz and Greg Moore for useful conversations. W. S. thanks the High Energy Group at Harvard for their kind hospitality.

\section{A Asymptotic constraints}

In this appendix we work out the asymptotic form of the constraint equations, which relate the leading order fluctuations of the metric. In a Dirac bracket formalism, the constraints, by construction, commute with everything. Therefore the generators of the ASG are ambiguous up to the additions of integrals proportional to the constraints.

The constraint equations are $G_{\mu}^{0}=0$. Using the boundary conditions (5.1), linearizing in $h_{\mu \nu}$ and expanding to leading order in $1 / r$, we can solve the asymptotic constraint equations as follows.

First consider $G_{\varphi}^{0}=0$. At leading order this is a second order differential equation for $h_{\varphi \varphi}$ in $\theta$, and does not involve the other metric components. The solution which leads to a metric regular at the poles is

$$
h_{\varphi \varphi}=\Lambda^{2} \Omega^{2} f(\tau, r, \varphi) .
$$

Now consider $G_{0}^{0}=0$. This is a function only of $\theta, h_{\tau \tau}, h_{\varphi \varphi}$, and their first and second $\theta$-derivatives. Plugging in the solution for $h_{\varphi \varphi}$, all the derivatives drop out and the solution is

$$
h_{\tau \tau}=r^{2}\left(1-\Lambda^{2}\right) \Omega^{2} f(\tau, r, \varphi)+O(r) .
$$

\footnotetext{
${ }^{12} \mathrm{~A}$ sufficient but not necessary condition for validity of the Cardy formula is $T>>c$. This condition is not obeyed here, as in many black hole applications [3]. In many such cases the formula is nevertheless valid because of the small gap arising from highly twisted sectors [54]. For example we might expect a twisted sector of order $J$, which is effectively described by a universal $c_{L}=12$ "long string" CFT at temperature $T_{L}=\frac{J}{2 \pi}$. A small gap is generic for black holes [55] so we hope that the same mechanism is operative here.
} 
Now consider $G_{\theta}^{0}=0$. This is proportional to

$$
2 \Lambda^{2}\left(\Lambda \partial_{\theta} \Omega-\Omega \partial_{\theta} \Lambda\right) h_{r \varphi}-\Lambda^{3} \Omega \partial_{\theta} h_{r \varphi}-\Omega \partial_{\theta} \Lambda \partial_{\varphi} h_{\varphi \varphi}
$$

Plugging in the solution above for the $\theta$-dependence of $h_{\varphi \varphi}$, we find

$$
h_{r \varphi}=-\frac{1}{r}\left(\frac{\Omega^{2}}{2} \partial_{\varphi} f(\tau, r, \varphi)+\frac{16 \Omega^{2}}{\Lambda^{2}} g(\tau, r, \varphi)\right)+O\left(\frac{1}{r^{2}}\right)
$$

Now consider $G_{r}^{0}=0$. This is a function of $\theta, h_{r \varphi}, \partial_{\theta} h_{t \varphi}, \partial_{\theta}^{2} h_{r \varphi}, \partial_{\varphi} h_{\varphi \varphi}, \partial_{\varphi} h_{\tau \tau}$. Plugging in the solutions for $h_{\mu \nu}$ from above, the final condition is $g(\tau, r, \varphi)=0$. Note that the constraints imply $h \equiv \bar{g}^{\mu \nu} h_{\mu \nu}=0$.

\section{B Charge integrability}

In this appendix we show that to quadratic order around the NHEK background, the charges (6.3) do not depend on the path of integration over metrics, $\gamma$. Since $E_{R}=0$, only $Q_{\zeta_{\epsilon}}[g]$ needs to be checked. The integrability condition is

$$
\int_{\partial \Sigma}\left(k_{\zeta_{\epsilon}}[h, g+\tilde{h}]-k_{\zeta_{\epsilon}}[\tilde{h}, g+h]-k_{\zeta_{\epsilon}}[h-\tilde{h}, g]\right)=0
$$

keeping terms up to order $h \tilde{h}$. The integrand is

$$
\begin{aligned}
& -\frac{1}{8} \epsilon_{\alpha \beta \mu \nu}\left[\tilde{h}\left(\zeta^{\nu} D^{\mu} h-\zeta^{\nu} D_{\sigma} h^{\mu \sigma}+\frac{1}{2} h^{\sigma \nu}\left(D^{\mu} \zeta_{\sigma}+D_{\sigma} \zeta^{\mu}\right)\right)+\zeta^{\nu} h^{\lambda \mu} D_{\lambda} \tilde{h}\right. \\
& -\zeta^{\nu}\left(2 D_{\sigma} \tilde{h}_{\lambda}^{\mu}-D^{\mu} \tilde{h}_{\lambda \sigma}\right) h^{\lambda \sigma}+\zeta^{\sigma} h^{\lambda \nu} D_{\sigma} \tilde{h}_{\lambda}^{\mu}-\left(h_{\sigma \lambda} \tilde{h}^{\nu \lambda} D^{\mu} \zeta^{\sigma}+h_{\sigma}^{\nu} \tilde{h}^{\mu \lambda} D_{\lambda} \zeta^{\sigma}\right) \\
& -(h \leftrightarrow \tilde{h})] d x^{\alpha} \wedge d x^{\beta}
\end{aligned}
$$

Using the boundary conditions (5.1) and the constraints $h=\tilde{h}=0$ derived in Appendix A, the component tangent to $\partial \Sigma$ vanishes on the NHEK background $\bar{g}_{\mu \nu}$ for $\zeta=\zeta_{\epsilon}$.

\section{References}

[1] J. Maldacena, "The large $\mathrm{N}$ limit of superconformal field theories and supergravity," Adv. Theor. Math. Phys. 2, 231 (1998) [Int. J. Theor. Phys. 38, 1113 (1998)] [hep-th/9711200].

[2] J. D. Brown and M. Henneaux, "Central Charges in the Canonical Realization of Asymptotic Symmetries: An Example from Three-Dimensional Gravity," Commun. Math. Phys. 104, 207 (1986). 
[3] A. Strominger and C. Vafa, "Microscopic Origin of the Bekenstein-Hawking Entropy," Phys. Lett. B 379, 99 (1996) hep-th/9601029].

[4] A. Strominger, "Black hole entropy from near-horizon microstates," JHEP 9802, 009 (1998) arXiv:hep-th/9712251.

[5] C. P. Herzog, A. Karch, P. Kovtun, C. Kozcaz and L. G. Yaffe, "Energy loss of a heavy quark moving through $\mathrm{N}=4$ supersymmetric Yang-Mills plasma," JHEP 0607, 013 (2006) arXiv:hep-th/0605158]. S. S. Gubser, "Drag force in AdS/CFT," Phys. Rev. D 74, 126005 (2006) arXiv:hep-th/0605182]. C. P. Herzog, "Energy loss of heavy quarks from asymptotically AdS geometries," JHEP 0609, 032 (2006) arXiv:hep-th/0605191]. J. J. Friess, S. S. Gubser and G. Michalogiorgakis, "Dissipation from a heavy quark moving through $\mathrm{N}=4$ super-Yang-Mills plasma," JHEP 0609, 072 (2006) arXiv:hep-th/0605292].

[6] H. Liu, K. Rajagopal and U. A. Wiedemann, "Wilson loops in heavy ion collisions and their calculation in AdS/CFT," JHEP 0703, 066 (2007) arXiv:hep-ph/0612168].

[7] D. T. Son and A. O. Starinets, "Viscosity, Black Holes, and Quantum Field Theory," Ann. Rev. Nucl. Part. Sci. 57, 95 (2007) [arXiv:0704.0240 [hep-th]].

[8] C. P. Herzog, P. Kovtun, S. Sachdev and D. T. Son, "Quantum critical transport, duality, and M-theory," Phys. Rev. D 75, 085020 (2007) arXiv:hep-th/0701036].

[9] S. A. Hartnoll, P. K. Kovtun, M. Muller and S. Sachdev, "Theory of the Nernst effect near quantum phase transitions in condensed matter, and in dyonic black holes," Phys. Rev. B 76, 144502 (2007) [arXiv:0706.3215 [cond-mat.str-el]].

[10] S. A. Hartnoll, C. P. Herzog and G. T. Horowitz, "Building a Holographic Superconductor," Phys. Rev. Lett. 101, 031601 (2008) [arXiv:0803.3295 [hep-th]].

[11] K. Balasubramanian and J. McGreevy, "Gravity duals for non-relativistic CFTs," Phys. Rev. Lett. 101, 061601 (2008) arXiv:0804.4053 [hep-th]].

[12] D. T. Son, "Toward an AdS/cold atoms correspondence: a geometric realization of the Schroedinger symmetry," Phys. Rev. D 78, 046003 (2008) [arXiv:0804.3972 [hep-th]].

[13] J. E. McClintock, R. Shafee, R. Narayan, R. A. Remillard, S. W. Davis and L. X. Li, "The Spin of the Near-Extreme Kerr Black Hole GRS 1915+105," Astrophys. J. 652, 518 (2006) arXiv:astro-ph/0606076. 
[14] J. M. Maldacena and A. Strominger, "Universal low-energy dynamics for rotating black holes," Phys. Rev. D 56, 4975 (1997) arXiv:hep-th/9702015.

[15] J. M. Maldacena and A. Strominger, "Black hole greybody factors and D-brane spectroscopy," Phys. Rev. D 55, 861 (1997) arXiv:hep-th/9609026.

[16] R. A. Remillard and J. E. McClintock, "X-ray Properties of Black-Hole Binaries," Ann. Rev. Astron. Astrophys. 44, 49 (2006) arXiv:astro-ph/0606352.

[17] See for example the Kerrfest proceedings http://www.phys.canterbury.ac.nz/kerrfest.

[18] J. M. Bardeen and G. T. Horowitz, "The extreme Kerr throat geometry: A vacuum analog of $\operatorname{AdS}(2)$ x S(2)," Phys. Rev. D 60, 104030 (1999) arXiv:hep-th/9905099.

[19] M. Cvetic and F. Larsen, Phys. Rev. Lett. 82, 484 (1999) arXiv:hep-th/9805146.

[20] A. Dabholkar, A. Sen and S. P. Trivedi, "Black hole microstates and attractor without supersymmetry," JHEP 0701, 096 (2007) arXiv:hep-th/0611143.

[21] G. T. Horowitz and M. M. Roberts, "Counting the Microstates of a Kerr Black Hole," Phys. Rev. Lett. 99, 221601 (2007) arXiv:0708.1346 [hep-th]].

[22] R. P. Kerr, "Gravitational field of a spinning mass as an example of algebraically special metrics," Phys. Rev. Lett. 11, 26 (1963).

[23] M. Visser, "The Kerr spacetime: A brief introduction," arXiv:0706.0622 [gr-qc].

[24] J. D. Bekenstein, "Black Holes And Entropy," Phys. Rev. D 7, 2333 (1973).

[25] S. W. Hawking, "Particle Creation By Black Holes," Commun. Math. Phys. 43, 199 (1975).

[26] S. Detournay, D. Orlando, P. M. Petropoulos and P. Spindel, "Three-dimensional black holes from deformed anti de Sitter," JHEP 0507, 072 (2005) arXiv:hep-th/0504231.

[27] I. Bengtsson and P. Sandin, "Anti-de Sitter space, squashed and stretched," Class. Quant. Grav. 23, 971 (2006) arXiv:gr-qc/0509076.

[28] D. Anninos, W. Li, M. Padi, W. Song and A. Strominger, "Warped $A d S_{3}$ Black Holes," arXiv:0807.3040 [hep-th]. 
[29] H. Bondi, M. G. J. van der Burg and A. W. K. Metzner, "Gravitational waves in general relativity. 7. Waves from axisymmetric isolated systems," Proc. Roy. Soc. Lond. A 269, 21 (1962). R. K. Sachs, "Gravitational waves in general relativity. 8. Waves in asymptotically flat space-times," Proc. Roy. Soc. Lond. A 270, 103 (1962).

[30] G. Barnich and G. Compere, "Classical central extension for asymptotic symmetries at null infinity in three spacetime dimensions," Class. Quant. Grav. 24, F15 (2007) [Erratum-ibid. 24, 3139 (2007)] arXiv:gr-qc/0610130].

[31] G. Compere and S. Detournay, "Centrally extended symmetry algebra of asymptotically Goedel spacetimes," JHEP 0703, 098 (2007) arXiv:hep-th/0701039.

[32] G. Compere and S. Detournay, "Semi-classical central charge in topologically massive gravity," arXiv:0808.1911 [hep-th].

[33] M. Guica and A. Strominger, "Wrapped M2/M5 duality," arXiv:hep-th/0701011.

[34] G. Barnich and F. Brandt, "Covariant theory of asymptotic symmetries, conservation laws and central charges," Nucl. Phys. B 633, 3 (2002) arXiv:hep-th/0111246.

[35] G. Barnich and G. Compere, "Surface charge algebra in gauge theories and thermodynamic integrability," J. Math. Phys. 49, 042901 (2008) [arXiv:0708.2378 [gr-qc]].

[36] L. F. Abbott and S. Deser, "Stability Of Gravity With A Cosmological Constant," Nucl. Phys. B 195, 76 (1982).

[37] V. Iyer and R. M. Wald, "Some properties of Noether charge and a proposal for dynamical black hole entropy," Phys. Rev. D 50, 846 (1994) arXiv:gr-qc/9403028.

[38] I. M. Anderson and C. G. Torre, "Asymptotic conservation laws in field theory," Phys. Rev. Lett. 77, 4109 (1996) arXiv:hep-th/9608008.

[39] C. G. Torre, "Local cohomology in field theory with applications to the Einstein equations," arXiv:hep-th/9706092.

[40] G. Barnich, F. Brandt and M. Henneaux, "Local Brst Cohomology In The Antifield Formalism. 1. General Theorems," Commun. Math. Phys. 174, 57 (1995) arXiv:hep-th/9405109.

[41] G. Barnich, F. Brandt and M. Henneaux, "Local BRST cohomology in gauge theories," Phys. Rept. 338, 439 (2000) arXiv:hep-th/0002245. 
[42] G. Barnich, "Boundary charges in gauge theories: Using Stokes theorem in the bulk," Class. Quant. Grav. 20, 3685 (2003) arXiv:hep-th/0301039].

[43] G. Compere, "Symmetries and conservation laws in Lagrangian gauge theories with applications to the mechanics of black holes and to gravity in three dimensions," arXiv:0708.3153 [hep-th].

[44] T. Regge and C. Teitelboim, "Role Of Surface Integrals In The Hamiltonian Formulation Of General Relativity," Annals Phys. 88, 286 (1974).

[45] G. Barnich, F. Brandt and K. Claes, "Asymptotically anti-de Sitter space-times: symmetries and conservation laws revisited," Nucl. Phys. Proc. Suppl. 127, 114 (2004) arXiv:gr-qc/0306112.

[46] J. D. Brown and J. W. . York, "Quasilocal energy and conserved charges derived from the gravitational action," Phys. Rev. D 47, 1407 (1993) arXiv:gr-qc/9209012.

[47] V. Balasubramanian and P. Kraus, "A stress tensor for anti-de Sitter gravity," Commun. Math. Phys. 208, 413 (1999) hep-th/9902121.

[48] M. Banados, G. Barnich, G. Compere and A. Gomberoff, "Three dimensional origin of Goedel spacetimes and black holes," Phys. Rev. D 73, 044006 (2006) arXiv:hep-th/0512105.

[49] G. Barnich and G. Compere, "Conserved charges and thermodynamics of the spinning Goedel black hole," Phys. Rev. Lett. 95, 031302 (2005) arXiv:hep-th/0501102.

[50] G. Duffy and A. C. Ottewill, "The renormalized stress tensor in Kerr space-time: Numerical results for the Hartle-Hawking vacuum," Phys. Rev. D 77, 024007 (2008) arXiv:gr-qc/0507116.

[51] V. P. Frolov and K. S. Thorne, "Renormalized Stress-Energy Tensor Near the Horizon of a Slowly Evolving, Rotating Black Hole" Phys. Rev. D 39, 2125 (1989).

[52] A. C. Ottewill and E. Winstanley, "The renormalized stress tensor in Kerr space-time: General results," Phys. Rev. D 62, 084018 (2000) arXiv:gr-qc/0004022.

[53] J. M. Maldacena and A. Strominger, "AdS(3) black holes and a stringy exclusion principle," JHEP 9812, 005 (1998) arXiv:hep-th/9804085.

[54] J. M. Maldacena and L. Susskind, "D-branes and Fat Black Holes," Nucl. Phys. B 475, 679 (1996) arXiv:hep-th/9604042. 
[55] J. Preskill, P. Schwarz, A. D. Shapere, S. Trivedi and F. Wilczek, "Limitations on the statistical description of black holes," Mod. Phys. Lett. A 6, 2353 (1991). 